\title{
Boundary Layer Flow Over a Vertical Cylinder Embedded in a Porous Medium Moving with non Linear Velocity
}

\author{
Tarek G Emam \\ Department of Mathematics, College of Science and Arts-Khulais \\ University of Jeddah, \\ Jeddah, \\ SAUDI ARABIA. \\ and \\ Department of Mathematics, Faculty of Science, \\ Ain Shams University, \\ Cairo, \\ EGYPT.
}

\begin{abstract}
In this work, we consider a steady flow of an incompressible fluid over a cylinder which is semi-infinite. The cylinder is embedded in a porous medium and is considered to move vertically with nonlinear velocity. A system of ordinary differential equations is obtained from the partial differential equations governing the motion using a self-similarity transformation. Such system of ordinary differential equations is solved numerically after obtaining the missed initial conditions. The problem has been solved analytically for the linear velocity case. Numerical and analytical results for such case are given to validate the numerical method used.
\end{abstract}

Key-Words: - Boundary Layer Flow, Porous Medium, Moving Cylinder, Nonlinear Velocity, Similarity Solution, Numerical solution. )

Received: August 31, 2020. Revised: February 7, 2021. Accepted: February 19, 2021. Published: March 4, 2021.

\section{Introduction}

Many applications in industry and engineering arise from the flow of Newtonian and NonNewtonian fluids. Authors have been interested in such fields for the last few decades. The problem of boundary layer flow over a cylinder, which is our concern in this work, has many applications such as glass fiber production, the drawing of wires, as well as plastic and metallurgy industries. Sakiadis [1] was the first to study boundary layer flow behavior on a cylinder moving in a Newtonian fluid. He obtained a numerical solution using a similarity transformation. Rotte and Beek have given Some models for the calculation of heat transfer coefficients to a moving continuous cylinder[2]. Ganesan and Loganathan [3] have introduced the problem of radiation and mass transfer effects on flow of an incompressible viscous fluid past a moving vertical cylinder. Recently Ado-Eldahab and Salem [4] have studied the flow and heat transfer of non-Newtonian powerlaw fluid with diffusion and chemical reaction on a moving cylinder. Amkadni and Azzouzi [5] have analyzed the steady flow of an incompressible electrically conducting fluid over a semi-infinite moving vertical cylinder in the presence of a uniform transverse magnetic field. The Study of hydrodynamic flow and the transfer of heat in a porous medium is very interesting for its wide range of applications on the process of controlling boundary layer flow such as the removal of heat from nuclear debris. Elbashbeshy et al [6] have analyzed the problem of boundary layer flow over a stretching horizontal cylinder embedded in a porous medium. They have considered the effects of thermal radiation, heat transfer, and suction/injection. Abdul Rehman et al [7] have given an analytic solution to the problem of, axisymmetric Stagnation Flow of a Micropolar Fluid in a Moving Cylinder. Haroon et al [8] have presented an investigation provides an view in the steady, incompressible and electrically conducting boundary layer flow of viscoelastic nanofluid flowing due to a moving, linearly stretched surface. More recent works discussing the boundary layer flow over a cylinder can be found in the references[9]-[14] In this paper we present a solution to the problem of a vertically moving cylinder with nonlinear velocity. Analytic solution is given for the case of linear velocity and a comparison between analytic and numerical solutions is given 
in some cases to validate the numerical method used in this paper. Considering nonlinear form of the cylinder velocity enables us to be closer to the real problem of boundary layer flow over a moving cylinder and suggests new problems such as the study of heat transfer over a moving cylinder with nonlinear velocity.

\section{Problem Formulation}

Consider a steady incompressible laminar flow past a moving cylinder. The cylinder is assumed to be semi-infinite and vertical with radius $R$. A uniform transverse magnetic filed with strength $B_{0}$ is applied. We also assume that the fluid properties are constant. The induced magnetic field is neglected since we consider that the Reynolds number is very small. Along the axis of the cylinder we measure the axial coordinate $x$ while the radial coordinate $r$ is measured normal to the axis of the cylinder. The external velocity is taken in the form $u_{e}(x)=u_{\infty}\left(\frac{x}{l}\right)^{n}$, where $u_{\infty}>0$. Such assumptions along with the boundary layer approximation results in the following governing equations:

$$
\begin{gathered}
\frac{\partial r u}{\partial x}+\frac{\partial r v}{\partial r}=0 \\
u \frac{\partial u}{\partial x}+v \frac{\partial u}{\partial r}=\frac{\nu}{r} \frac{\partial}{\partial r}\left(r \frac{\partial u}{\partial r}\right)+u_{e} \frac{d u_{e}}{d x}+\frac{\nu}{\kappa_{p}}\left(u_{e}-u\right)
\end{gathered}
$$

subject to the conditions:

$$
\begin{array}{r}
u(R, x)=u_{w}\left(\frac{x}{l}\right)^{n}, v(R, x)=0, \\
\lim _{r \rightarrow \infty} u(r, x)=u_{e}\left(\frac{x}{l}\right)
\end{array}
$$

where $u$ and $v$ are the velocity components along the directions of $x$ and $r$ respectively, $\nu$ is the kinematic viscosity, $\rho$ is the fluid density, and $\sigma$ is the electrical conductivity of the fluid, $l$ is the characteristic length

and $\kappa_{p}$ is the porosity of the medium. We define the stream function $\psi$ as

$$
r u=\frac{\partial \psi}{\partial r}, \quad r v=-\frac{\partial \psi}{\partial x}
$$

Substituting from equation (4) into equations (1) and (2) we find that equation (1) is satisfied identically and equation (2) takes the form:

$$
\begin{gathered}
\frac{1}{r^{2}} \frac{\partial \psi}{\partial r} \frac{\partial^{2} \psi}{\partial x \partial r}+\frac{1}{r^{3}} \frac{\partial \psi}{\partial x} \frac{\partial \psi}{\partial r}-\frac{1}{r^{2}} \frac{\partial \psi}{\partial x} \frac{\partial^{2} \psi}{\partial r^{2}}=\frac{\nu}{r^{3}} \frac{\partial \psi}{\partial r} \\
-\frac{\nu}{r^{2}} \frac{\partial^{2} \psi}{\partial r^{2}}+\frac{\nu}{r} \frac{\partial^{3} \psi}{\partial r^{3}}+u_{e} \frac{d u_{e}}{d x}+\frac{\nu}{\kappa_{p}}\left(u_{e}-\frac{1}{r} \frac{\partial \psi}{\partial r}\right)
\end{gathered}
$$

and the conditions (3) are transformed into the form:

$$
\begin{array}{r}
\frac{\partial \psi(R, x)}{\partial r}=R u_{w}\left(\frac{x}{l}\right)^{n}, \frac{\partial \psi(R, x)}{\partial x}=0, \\
\lim _{r \rightarrow \infty}\left(\frac{1}{r} \frac{\partial \psi(r, x)}{\partial r}\right)=u_{\infty}\left(\frac{x}{l}\right)^{n}
\end{array}
$$

we look for a solution in the form

$$
\begin{gathered}
\psi(r, x)=\sqrt{\frac{\nu u_{\infty} R(n+1)}{2}\left(\frac{x}{l}\right)^{n+1}} R f(\eta), \\
\eta=\sqrt{\frac{u_{\infty}}{2 \nu(n+1) R}\left(\frac{x}{l}\right)^{n-1}} \frac{1}{R}\left(r^{2}-R^{2}\right)
\end{gathered}
$$

where $f$ is the dimensionless stream function and $\eta$ is the similarity variable. Upon substituting in equations (5) and (6), we get:

$$
\begin{aligned}
& \quad \frac{2}{n+1}(\eta K+1) f^{\prime \prime \prime}(\eta)+\left(\frac{2 K}{n+1}+\frac{\epsilon(n+1)}{2} f(\eta)\right) f^{\prime \prime}(\eta) \\
& +\quad n \epsilon\left(1-f^{\prime 2}(\eta)\right)-\kappa\left(f^{\prime}(\eta)-1\right)=0, \\
& f(0)=0, \quad f^{\prime}(0)=\frac{u_{w}}{u_{\infty}}=a, \quad f^{\prime}(\infty)=1
\end{aligned}
$$

where $K=\sqrt{\frac{\nu(n+1)}{u_{\infty} R}\left(\frac{l}{x}\right)^{n-1}}, \epsilon=\frac{R}{l}$, and the permeability parameter is $\kappa=\frac{R \nu}{\kappa_{p} u_{\infty}}\left(\frac{l}{x}\right)^{n-1}$.

The system (8) - (9) is transformed into a system of first order differential equations through assuming that: $y_{1}=f, y_{2}=f^{\prime}, y_{3}=f^{\prime \prime}$ to get

$$
y_{1}^{\prime}(\eta)=y_{2}(\eta)
$$

$$
\begin{aligned}
& y_{2}^{\prime}(\eta)=y_{3}(\eta) \\
& y_{3}^{\prime}(\eta)=y_{2}(\eta) \\
& \quad \frac{2}{n+1}(K \eta+R) y_{3}^{\prime \prime}(\eta)=-\left(\frac{2 K}{n+1}+\frac{\epsilon(n+1)}{2} y_{1}(\eta)\right) \\
& \times \quad y_{2}(\eta)-n \epsilon\left(1-\left(y_{2}^{2}(\eta)\right)+\kappa\left(y_{2}(\eta)-1\right)\right.
\end{aligned}
$$

subject to the initial conditions:

$$
y_{1}(0)=0, \quad y_{2}(0)=a, \quad y_{3}(0)=s
$$

Suitable numerical values are given for $a, n, \kappa, \epsilon$, and $K$. the value of $s$ is priori unknown that is determined as part of the solution. We use Mathematica to define a function $F[s]=$ NDSolve[(10) - (14)]. The value of $s$ is obtained through solving the equation $y_{1}\left(\eta_{\max }\right)=1$. A suitable start value of $\eta$ is taken and then increased to reach $\eta_{\max }$ for which the difference between two successive values of $s$ is less than $10^{-7}$. So the problem now is an initial value problem which is then solved using NDSolve, see references[6] and [15]. 


\section{Problem Solution}

In this section, solutions of the problem are introduced. An analytic solution is given in the special case $n=1$, where the cylinder velocity is considered to be linear. Numerical solutions are given for the case $n \neq 1$.

\subsection{Special Case: $n=1$}

Here we consider the case $n=1$ which means that the cylinder moves with linear velocity. Equation (8) takes the form

$$
\begin{aligned}
& (\eta K+1) f^{\prime \prime \prime}(\eta)+(K+\epsilon f(\eta)) f^{\prime \prime}(\eta) \\
& +\epsilon\left(1-f^{\prime 2}(\eta)\right)-\kappa\left(f^{\prime}(\eta)-1\right)=0
\end{aligned}
$$

subject to the conditions:

$$
f(0)=0, \quad f^{\prime}(0)=\frac{u_{w}}{u_{\infty}}=a, \quad f^{\prime}(\infty)=1
$$

where $K=\sqrt{\frac{2 \nu}{u_{\infty} R}}$, and $\kappa=\frac{R \sigma B_{0}^{2}}{\rho u_{\infty}}$.

We seek for an exact solution to the linear case in the form

$$
f(\eta)=\eta+p+q e^{-\mu \eta}
$$

where $p, q, \mu$ are constants and $\mu>0$. So

$$
f^{\prime}(\eta)=1-q \mu e^{-\mu \eta}
$$

Applying the conditions $f(0)=0, f^{\prime}(0)=a$ we find that

$$
f(\eta)=\eta+\frac{a-1}{\mu}\left(1-e^{-\mu \eta}\right)
$$

the condition $f^{\prime}(\infty)=1$ is satisfied identically which can be observed from equation (18).

Substituting equation (19) into equation (15) we get the following:

$$
\left((\eta K+1) \mu^{2}-\mu K-\eta \epsilon \mu-2 \epsilon-\kappa\right)(a-1)-\epsilon(a-1)^{2}=0
$$

Using the fact that $a \neq 1$ and equating the coefficients of $\eta$ and $\eta^{0}$, we get

$$
\mu=\epsilon \sqrt{\frac{u_{\infty} R}{2 \nu}}=\frac{\epsilon}{K}
$$

and

$$
\kappa=\left(\frac{\epsilon}{K}\right)^{2}-\epsilon(a+2)
$$

hence the exact solution of equation (15) is

$$
f(\eta)=\eta+\frac{(a-1) k}{\epsilon}\left(1-e^{-\frac{\epsilon}{K} \eta}\right)
$$

where $\kappa$ is given by equation (22)

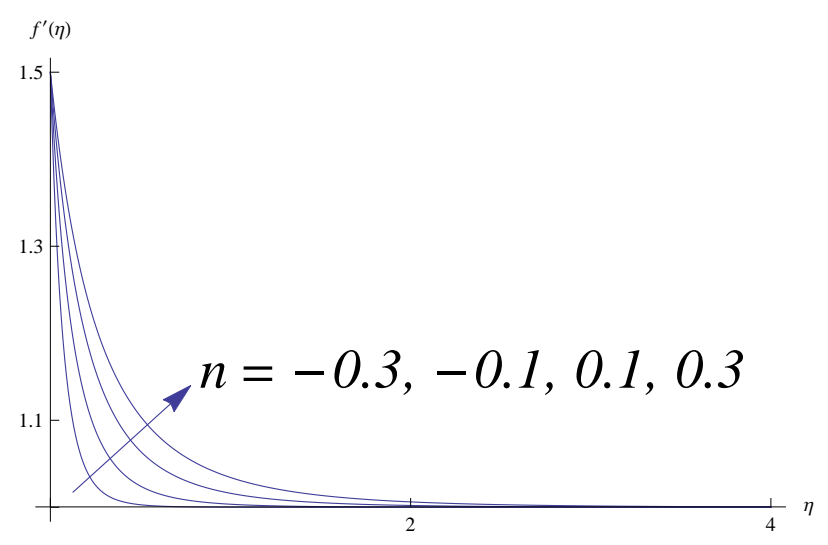

Figure 1: Variation of the fluid velocity with the nonlinearity parameter $n$, where $K=2, a=1.5$, $\epsilon=1$, and $\kappa=1$.

\subsection{Validation of the numerical method}

To validate the numerical method used in this paper we compare the numerical solution with the exact one for the case $n=1$. Table 1 gives a comparison between the values of $f^{\prime \prime}(0)$ for $K=2, \epsilon=1$ and different values of $a$

Table 1:Values of $f^{\prime \prime}(0)$, where $K=0.2, \epsilon=1$

\begin{tabular}{|c|c|c|c|}
\hline$a$ & Exact Soln. & Num. Soln. & Error: $\left|f^{\prime}\left(\eta_{\max }\right)-1\right|$ \\
\hline 1.2 & -1 & -1 & $9.77 \times 10^{-10}$ \\
1.5 & -2.5 & -2.5 & $2.45 \times 10^{-9}$ \\
2 & -5 & -5 & $2.38 \times 10^{-11}$ \\
3 & -10 & -10 & $4.32 \times 10^{-8}$ \\
\hline
\end{tabular}

The fourth column of Table 1 gives the numerical calculated values of $\left|f^{\prime}\left(\eta_{\max }\right)-1\right|$. Exact values should be zeros if $\eta_{\max } \rightarrow \infty$. The results shown in table 1 ensures that the numerical method used in this paper is valid.

\subsection{Case: $n \neq 1$}

Numerical solutions where $n \neq 1$ are obtained using the method described at the end of section 2 . The variations of the velocity $f^{\prime}(\eta)$ with the similarity variable $\eta$ are plotted for different values of the considered parameters. Figures (1)-(4) show that the fluid velocity $f^{\prime}(\eta)$ decreases with the increase of $\eta$ till it reaches the ambient fluid velocity that is $f^{\prime}(\eta)=1$. Figure 1 shows the variation of $f^{\prime}(\eta)$ for different values of the parameter $n$ where the other fluid parameters are kept constant. From the figure one can observe that as $n$ elevates the value of $f^{\prime}(\eta)$ increases. The physical justification of this behaviour is that since as $n$ increases the cylinder velocity increases which enforces the fluid velocity to increase which is clearly shown in the figure. The variation of the fluid velocity $f^{\prime}(\eta)$ with the permeability parameter $\kappa$ is shown in figure 2. To interpret the results shown 


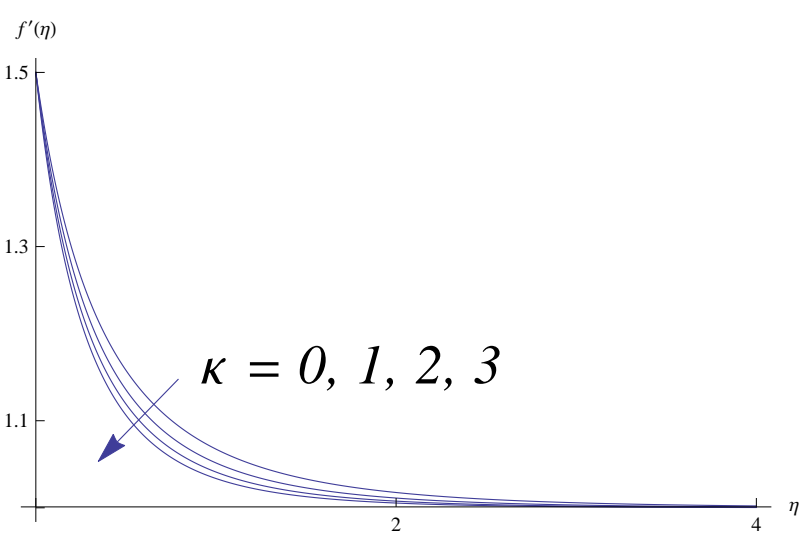

Figure 2: Variation of the fluid velocity with the permeability parameter $\kappa$, where $K=2, a=1.5$, $\epsilon=1$, and $n=0.5$.

in figure we know that $\kappa$ plays a considerable role in controlling the fluid velocity, since the value of $\kappa_{p}$ is a measure of how it is easy to penetrate the porous medium. $\kappa$ is the reciprocal of $\kappa_{p}$ so the increase of the permeability parameter $\kappa$ increases the resistance to the fluid motion and consequently the fluid velocity $f^{\prime}(\eta)$ decreases which coincides with the results shown in figure 2 .

The parameter $\epsilon$ has a considerable effect on the fluid velocity as depicted in figure 3. One can notice that the decrease of $\epsilon=\frac{R}{l}$ increases the fluid velocity. A reasonable explanation of this is that the decrease of $R$ results in decreasing the cylinder surface area, so the cylinder shrinks and as a result the space provided for the fluid free stream velocity increases. Thus the tendency of the fluid velocity to be a free stream is enhanced. Figure 4 ensures the fact that as the fluid velocity increases as the initial velocity increases.

\section{Conclusion}

An investigation of the problem of a boundary layer flow over a vertical cylinder moving with nonlinear velocity is given. An exact solution has been found for some special cases. Similarity solution of the problem has been given and the profiles of the fluid velocity have been plotted to show the variation of the fluid velocity with the considered parameters. The following results have been obtained:

- The fluid velocity increases as $n$, the parameter of nonlinearity increases and as the fluid initial velocity increases also.

- The fluid velocity decreases with the increase of the permeability parameter as well as the cylinder radius.

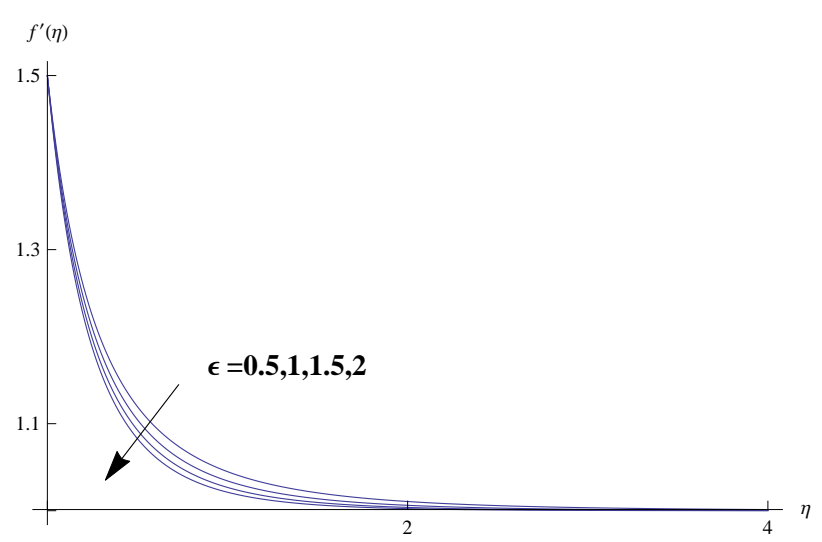

Figure 3: Variation of the fluid velocity with the parameter $\epsilon$, where $K=2, a=1.5, n=0.3$, and $\kappa=1$.

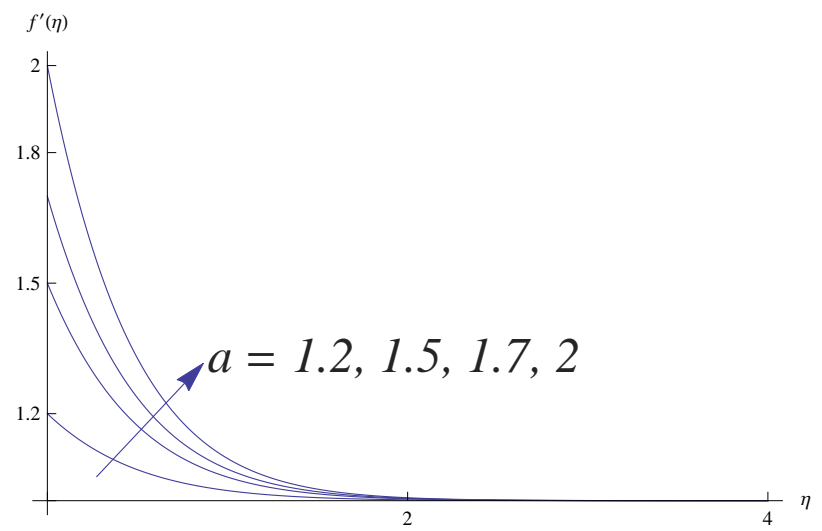

Figure 4: Variation of the fluid velocity with the initial velocity parameter, where $K=0.2, n=$ $0.3, \epsilon=1$, and $\kappa=1$. 


\section{References:}

[1] B. C. Sakiadis, Boundary-layer behavior on continuous solid surfaces: II. The boundary layer on a continuous flat surface, AIChE Journal 7, Nr. 2, 1961, pp. 221-225.

[2] J. W. Rotte and W. J. Beek, Some models for the calculation of heat transfer coefficients to a moving continuous cylinder, Chemical Engineering Science, 24, Nr. 4, 1969, pp. 705-716.

[3] P. Ganesan and P. Loganathan, Radiation and mass transfer effects on flow of an incompressible viscous fluid past a moving vertical cylinder, International Journal of Heat and Mass Transfer, 45, Nr. 21, 2002, pp. 4281-4288.

[4] E. M. Abo-Eldahab and A. M. Salem, MHD Flow and heat transfer of non-Newtonian power law fluid with diffusion and chemical reaction on a moving cylinder, Heat and Mass Transfer, 41, Nr. 8, 2005, pp. 703-708.

[5] M. Amkadni and A. Azzouzi, On a similarity solution of MHD boundary layer flow over a moving vertical cylinder, Differential Equations and Nonlinear Mechanics, Volume 2006, Article ID 52765, pp. 1-9.

[6] Elsayed M. A. Elbashbeshy, T. G. Emam, M. S. El-Azab, and K. M. Abdelgaber, Effect of thermal radiation on flow, heat, and mass transfere of a nanofluid over a stretching horizontal cylinder embedded in a porous medium with suction/injection, J. porous media, 18, Nr. 3, 2015, pp. 215-229.

[7] Abdul Rehman, Saleem Iqbal, Syed Mohsin Raza, Axisymmetric Stagnation Flow of a Micropolar Fluid in a Moving Cylinder: An Analytical Solution, Fluid Mechanics, 2, Nr. 1, 2016, pp. 1-7.

[8] Haroon Rasheed, Abdul Rehman, Naveed Sheikh, Saleem Iqbal. MHD Boundary Layer Flow of Nanofluid over a Continuously Moving Stretching Surface. Applied and Computational Mathematics. Vol. 6, Nr. 6, 2017, pp. 265-270.
[9] P. Loganathan1 and B. Eswari,Natural Convective Flow over moving Vertical Cylinder with Temperature Oscillations in the Presence of Porous Medium, Global Journal of Pure and Applied Mathematics, 13, Nr.2, 2017, pp. 839855 .

[10] P. T. Manjunatha1, · B. J. Gireesha, and B. C. Prasannakumara, Effect of Radiation on Flow and Heat Transfer of MHD Dusty Fluid Over a Stretching Cylinder Embedded in a Porous Medium in Presence of Heat Source, Int. J. Appl. Comput. Math, 3, 2017, pp. 293310 .

[11] Shahirah Abu Bakar, Norihan Md Arifin, Fadzilah Md Ali Norfifah Bachok, Roslinda Nazar, and Ioan Pop, A Stability Analysis on Mixed Convection Boundary Layer Flow along a Permeable Vertical Cylinder in a Porous Medium Filled with a Nanofluid and Thermal Radiation, Applied Sciences, 8, Nr. 4, 2018, pp. 1-13.

[12] Matteo D. L. Dalla Vedova, and Parid Alimhillaj Novel Fluid Dynamic Nonlinear Numerical Models of Servovalves for Aerospace International Journal of Mechanics, pp. 39-51, Volume 13, 2019

[13] Mohamed S. ABDEL-WAHED, and Essam M. EL-SAID, MHD Flow and Heat Transfer Over a Moving Cylinder in a Nanofluid Under Convective boundary conditions and Heat Generation, Thermal Science, 23, Nr. 6, 2019, pp. 3785-3796.

[14] M.P.Mkhatshwa, S.S. Motsa, M.S.Ayano, and P. Sibanda, MHD mixed convective nanofluid flow about a vertical slender cylinder using overlapping multi-domain spectral collocation approach, Case Studies in Theremal Engineering, 18, 2020, pp. 1-12.

[15] Elsayed M. A. ELBASHBESHY, Tarek G. EMAM, Magdi S. El-AZAB, and Khaled M. ABDELGABER, Slip Effects On Flow, Heat, and Mass Transfer of Nanofluid over Stretching Horizontal Cylinder in the Presence of Suction/Injection, 20, Nr. 6, 2016, pp. 1813-1824.

\section{Creative Commons Attribution License 4.0} (Attribution 4.0 International, CC BY 4.0)

This article is published under the terms of the Creative Commons Attribution License 4.0 https://creativecommons.org/licenses/by/4.0/deed.en US 